\title{
A Comparison of Bacterial Composition in Diabetic Ulcers and Contralateral Intact Skin
}

\author{
Viktoria Gontcharova ${ }^{1}$, Eunseog Youn ${ }^{3}$, Yan $\operatorname{Sun}^{1}$, Randall D Wolcott ${ }^{2}$ and Scot E. Dowd ${ }^{1,4, *}$ \\ ${ }^{1}$ Medical Biofilm Research Institute, 4321 Marsha Sharp Freeway, Lubbock, TX 79407 \\ ${ }^{2}$ Southwest Regional Wound Care Clinic, 2002 Oxford Ave, Lubbock TX 79410 \\ ${ }^{3}$ Texas Tech University, Computer Science Department, P.O. Box 43104, Lubbock, TX 79409-3104 \\ ${ }^{4}$ Research and Testing Laboratory, Lubbock, TX
}

\begin{abstract}
An extensive portion of the healthcare budget is allocated to chronic human infection. Chronic wounds in particular are a major contributor to this financial burden. Little is known about the types of bacteria which may contribute to the chronicity, biofilm and overall bioburden of the wound itself. In this study we compare the bacteriology of wounds and associated intact skin. Wound and paired intact skin swabs (from a contralateral location) were collected. The bacterial diversity was determined using bacterial Tag-encoded FLX amplicon pyrosequencing (bTEFAP). Diversity analysis showed intact skin to be significantly more diverse than wounds on both the species and genus levels $(3 \%$ and $5 \%$ divergence). Furthermore, wounds show heightened levels of anaerobic bacteria, like Peptoniphilus, Finegoldia, and Anaerococcus, and other detrimental genera such as Corynebacterium and Staphylococcus. Although some of these and other bacterial genera were found to be common between intact skin and wounds, notable opportunistic wound pathogens were found at lower levels in intact skin. Principal Component Analysis demonstrated a clear separability of the two groups. The findings of the study not only greatly support the hypothesis of differing bacterial composition of intact skin and wounds, but also contribute additional insight into the ecology of skin and wound microflora. The increased diversity and lowered levels of opportunistic pathogens found in skin make the system highly distinguishable from wounds.
\end{abstract}

Keywords: Biofilm, skin, bacteria, diversity, pyrosequencing, chronic wounds, bTEFAP, microbiome.

\section{INTRODUCTION}

A major and ever increasing portion of the healthcare budget is dedicated to treatment of chronic infections [1]. These infections include chronic wounds, and comprise $60-80 \%$ of all human infectious diseases [2]. Chronic wounds, by definition are wounds that have a biological or physiological reason for not healing. One of the primarily barriers to healing has now been identified as biofilm phenotype polymicrobial infections [1-3]. Biofilm is the natural physiological state of bacteria and are typically defined as polymicrobial populations of cells encased in hydrated extracellular polymeric substances and attached to a surface (e.g. tissue). Bacteria found in chronic wounds, therefore, usually exist as biofilm communities [4-7]. Due to the physiological properties of biofilm phenotype, bacteria comprising the communities become highly resistant to many traditional therapies, thus one of the most successful strategies for the management of the wound is biofilm-based wound care [3-8].

Diabetic ulcers, a subgroup of chronic wounds, occur in about $15 \%$ of diabetic patients and often lead to hospitalization and amputation [9]. More than 80,000 amputations

*Address correspondence to this author at the Research and Testing Laboratory, 4321 Marsha Sharp Fwy., Lubbock, TX 79407, USA;

Tel: 806-789-6879; E-mail: sdowd@pathogenresearch.org occur in the US within the diabetic population per year and account for a threefold increase in risk of death within 18 months [10]. Major limb amputation is also associated with depression and increased mortality and morbidity rates [11, 12]. Thus, preventing amputation must be the primary ethical goal of wound care practitioners.

Little is known about the types of bacteria that might contribute to the bioburden in chronic wounds. Several recent studies are now shedding new light on the bacterial populations associated with chronic wounds [4, 13, 14], however there is not much information comparing the bacterial diversity of intact skin to that found in chronic wound biofilms. The medical and research communities have realized the diversity and composition of the chronic wounds may be an important influence to the chronicity of the wounds, thus it is important to assess the composition of diabetic ulcers and identify the differentiating qualities between intact skin and the wounds.

Only about $2 \%$ of all known bacteria are able to be cultured in the laboratory [15]. Molecular methods allow for chronic wounds and skin samples to be evaluated without the need for culture. Molecular methods are now available which will allow the patient to be diagnosed, treated and reevaluated in an appropriate timeframe while identifying a wider range of bacteria contributing to the biofilm population $[14,16]$. This is an important concept because newer molecular methods can identify even previously uncharacter- 
ized bacteria which are difficult or impossible to culture in the laboratory [13].

Using pyrosequencing 16s ribosomal DNA methods, we assessed the bacterial microflora of diabetic ulcers and intact skin. During this study we sought to characterize the microbiota consistently of chronic wounds (likely contributing to their chronicity) and contrast this with the microbiota of intact skin on the same subjects providing a comparative look at wound and skin microflora within the same individual. This could possibly provide insight into the importance of the diabetic condition and the possibility of the disease itself making a person vulnerable to specific types of chronic wound infections promoted by the subjects own skin.

\section{MATERIALS AND METHODS}

\section{Intact Skin and Wound Samples}

Debridement and skin swab samples were collected for 53 total samples (23 samples were collected from wounds and 28 from intact skin) at the Southwest Regional Wound Care Center in accordance with Western Institutional Review Board protocol number 20062347. All patients provided written consent. Diabetic subjects with chronic wounds were chosen for this study. All samples were collected using sterile technique and immediately frozen in collection tubes at $80^{\circ} \mathrm{C}$ until DNA extraction was performed as described previously [13]. Paired samples were collected from collateral locations on each subject of each other (ex. wound sample collected from left ankle, intact skin sample collected from same location on right ankle).

\section{Wound Sample DNA Extraction and bTEFAP}

DNA extractions and the bacterial tag-encoded FLX amplicon pyrosequencing (bTEFAP) were performed by Research and Testing Laboratories (Lubbock, TX; www. researchandtesting.com). Data from the study was deposited in the short read archive of NCBI.

\section{bTEFAP Sequence Processing Pipeline}

Scripts written in C\# within a Microsoft ${ }^{\circledR}$.NET (Microsoft Corp, Seattle, WA) development environment were used for all post sequencing processing. Discussion of software code is outside the scope of this report; however, a brief description of the algorithm follows. Quality trimmed sequences obtained from the FLX sequencing run were derived directly from FLX sequencing run output files. Tags were extracted from the multi-FASTA file into individual samplespecific files based upon the tag sequence. Tags which did not have $100 \%$ homology to the sample designation were not considered. Sequences which were less than $200 \mathrm{bp}$ after quality trimming were not considered. After parsing the tags into individual FASTA files the resultant individual samples were labeled and concatenated into one file containing all samples. The resulting FASTA formatted file was then chimera checked using a custom algorithm appropriate for high throughput analysis for the $16 \mathrm{~S}$ database available at http://www.researchandtesting.com/B2C2. Chimeric sequences were removed from the FASTA file to form a final FASTA sequence file. The final file was then evaluated using BLASTn [17] against a database derived from GenBank (http://ncbi.nlm.nih.gov). A post processing algorithm generated best-hit files. The parameters used have been previously evaluated to enable reliable identification at the genus level. Following best-hit processing a secondary post-processing algorithm was used to determine the percent composition per sample.

\section{Statistics}

Statistical analysis was performed on percent composition files. Further custom scripts were built to find the most discriminating and the least discriminating bacteria in each taxonomic level between intact and wound samples. Each bacterium was considered as a feature and applied a feature selection (ranking) method. We used the area under the ROC curve (AUC) [18, 19] feature selection method [20]. The analysis focused on the genus and species classifications. For each feature (taxonomic classification in this case), AUC was computed. This area under the curve represents the discrimination capability of the feature. The scores are scaled to $[0.5,1]$ to consider only the magnitude, not the direction of discrimination. Scores closest to 1 are more discriminating while those close to 0.5 represent bacteria found equally abundantly in both intact and wound samples.

The similarity between the compositions within various groups was performed using the Pearson correlation distances [21,22]. This evaluation was performed for distances between intact and wound samples, within wound samples, within intact samples, and between paired samples of intact and wounded skin on an individual.

To assess the separability of the intact skin and wound samples, Principal Component Analysis (PCA) was implemented. Custom scripts tailored for next generation data (Research and Testing Laboratory, Lubbock, TX) were implemented to formulate the environment and NEXUS tree files for a distance matrix based on the UNIFRAC [23] formula. This distance matrix was used for Principal Component Analysis and Hierarchical clustering implemented using Python scripts. Samples more similar to each other should appear closer together according to the respective axis reflecting the variation among all samples. This technique is useful in displaying clusters existing within data.

\section{RESULTS}

\section{Diversity Analysis}

Our group has previously published several research articles on the application of the bTEFAP pyrosequencing approach for the analysis of chronic wound microbiota $[7,13$, 14]. In the first paper, a broad survey of wounds analyzed by molecular methods indicated a highly diverse composition found in chronic wound samples. While the analysis resulted in interesting findings, the follow up analysis used individual samples instead of a pooled composition from multiple diabetic foot ulcers. The work indicated the absence of any single or discrete individual populations of bacteria acting as the culprit in chronic infections. No unique genus of bacteria was found in all samples, supporting the idea of functionally equivalent pathogroups establishing a highly resistant opportunistically pathogenic biofilm [24], contributing to the chronicity of the wound.

Continuing the molecular approach to chronic wound analysis, our group has now performed the comparison of 
chronic wounds to intact skin. It is the next logical step to not only identifying the major players in wounds, but also identifying the reasons for the intact collateral skin remaining healthy.

Pyrosequencing using the bTEFAP approach based upon titanium chemistry (average read length of $400 \mathrm{bp}$ ) produced a total of 148,349 sequences between the intact and wound samples. After quality scoring and eliminating sequences $<200$ nucleotides long, 101,950 sequences were used as the next set for analyses. Chimeric sequences represented $\sim 9 \%$ of the total samples and were removed leaving over 93,000 high quality sequence reads among samples. Microbial diversity analysis [25-28] was performed by clustering sequence tags into groups of defined sequence variation ranging from unique sequences to $10 \%$ divergence using DOTUR [29] as previously described [30]. Clusters acting as OTUs were used as input for calculations with the abundance-based coverage estimator ACE and the Chaol [31] estimator of species diversity and richness. Tables $\mathbf{1}$ and $\mathbf{2}$ show the microbial diversity estimates obtained with parametric and non-parametric modeling of rarefaction, ACE and
Chaol for intact skin and for samples extracted from wounds. When relatively large genetic distances $(5 \%$ divergence) are considered these estimates predict that even at the genus level there is significantly less diversity and richness of microbial communities in wounds. As sequence divergence is more stringently analyzed, the significance levels of diversity decrease but remains significant. At the species level (3\% divergence), wounds remain less diverse than the intact skin, according to all three approaches, Rarefaction, ACE, and Chao1. Table 3 summarizes the data from Tables $\mathbf{1}$ and $\mathbf{2}$ with average values from the data and indicates statistical significance between the intact skin samples. Divergence of $3 \%$ and $5 \%$ is indicative of sequences differing at the species and genus level, respectively. The student's t-test results seen in Table $\mathbf{3}$ indicate significantly higher diversity levels in intact skin when compared to wounds.

\section{Composition Analysis}

To better perform group comparisons, after the bTEFAP pipeline, the resulting files containing the percent bacterial composition per sample were grouped into "intact" and

Table 1. Diversity and Richness Data for Wound Samples

\begin{tabular}{|c|c|c|c|c|c|c|c|}
\hline 1 & 3965 & 104 & 61 & 139 & 70 & 137 & 67 \\
\hline 5 & 1653 & 54 & 38 & 91 & 65 & 92 & 67 \\
\hline 9 & 3896 & 47 & 31 & 71 & 70 & 67 & 51 \\
\hline 10 & 3340 & 101 & 57 & 155 & 86 & 153 & 87 \\
\hline 11 & 2734 & 100 & 61 & 142 & 80 & 138 & 101 \\
\hline 17 & 3167 & 76 & 46 & 114 & 76 & 129 & 81 \\
\hline 19 & 2010 & 67 & 45 & 97 & 70 & 99 & 64 \\
\hline 20 & 1462 & 39 & 25 & 66 & 42 & 71 & 36 \\
\hline 21 & 3256 & 35 & 17 & 48 & 33 & 44 & 31 \\
\hline 22 & 1298 & 109 & 81 & 150 & 103 & 133 & 95 \\
\hline 23 & 2884 & 153 & 98 & 238 & 135 & 254 & 141 \\
\hline 25 & 2259 & 135 & 81 & 167 & 91 & 169 & 90 \\
\hline
\end{tabular}

This table presents data at the $3 \%$ divergence level (corresponding to the species level) and the $5 \%$ divergence level (corresponding to the genus level). Four samples with a low number of reads were removed from the analysis. 
Table 2. Diversity and Richness Data for Intact Skin Samples

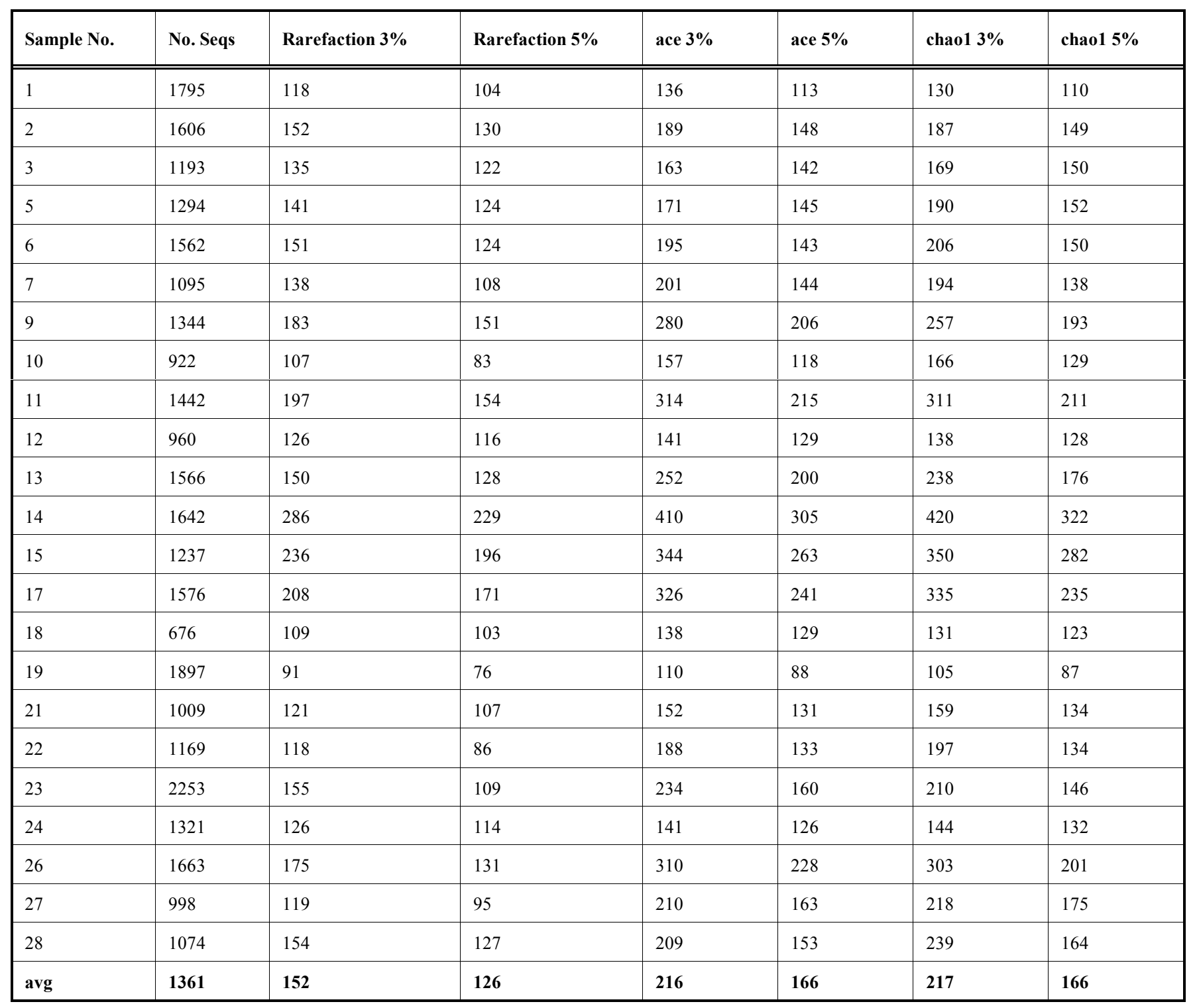

Data presented at the $3 \%$ divergence level (corresponding to the species level) and the $5 \%$ divergence level (corresponding to the genus level). Three samples with a low number of reads were removed from the analysis.

Table 3. P-values (P-val) Corresponding to a Student T-Test Evaluation of Intact Skin and Wound Samples

\begin{tabular}{|c|c|c|c|c|c|c|c|}
\hline$P$-val & $1.56 E-09$ & $4.40 E-06$ & $1.47 E-09$ & $4.62 E-05$ & $3.49 E-08$ & $4.82 E-05$ & $1.06 E-07$ \\
\hline Wound Avg & 2806 & 84 & 51 & 121 & 75 & 121 & 74 \\
\hline
\end{tabular}

"wound" groups. This data was analyzed using Area Under the Curve (AUC) $[18,19]$ analysis to identify the important genera within each group. Bacterial genera occurring in at least 20 of the samples (combination of intact and wounded regions) were considered to ensure a representative result and not attest to genera rarely found in the samples. Table 4 displays the most and the least discriminating bacteria from the samples along with the average percentages at which the bacteria were found in intact skin and wounds, Hmean and Wmean, respectively. An AUC value closest to 1 identifies a genus able to separate the wound and intact skin samples the best. AUC values closest to 0.5 represent genera least able to separate intact and wound samples whereas values closer to 1 are the most discriminating. These low AUC scores represent bacteria generally found at low amounts or similar levels in both groups of samples. 
Table 4. Most and Least Discriminating Genera

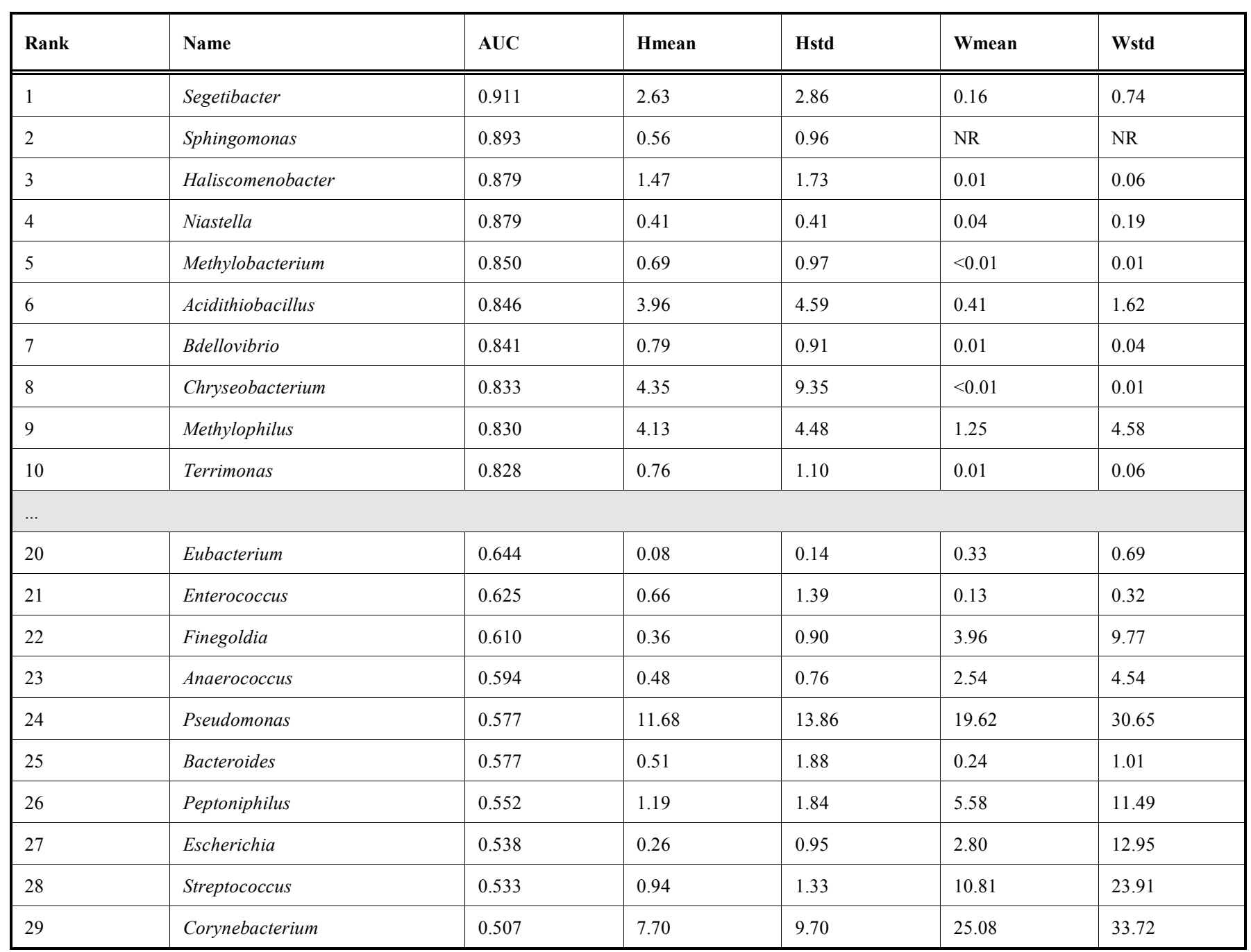

Table presents the Area Under the Curve, with values closest to 1 representing the most discriminating genera. The mean and standard deviation values for intact skin and wounds are presented in respective columns with "H" signifying intact (healthy) skin and "W" signifying wounds."NR" indicates a "not recovered" value.

The AUC approach produces synonymous results to the student's t-test. Both types of analyses address the question of significant differences among groups. The AUC approach was selected to provide a more intuitive and statistically relevant representation of the significance levels. The bacteria listed in Table 4, although prove to be highly distinguishing genera among intact skin and wound samples, seem to represent relatively low composition percentages within samples. However, even at low percentages, certain bacteria may play a significant role within the detrimental biofilm contributing to the overall ecosystem. Furthermore some of the least discriminating bacteria, such as E. coli, are significant contributors to bacterial environments with noticeably differing levels between the intact skin and wounds. The heightened levels evidently seem to play a role in distinguishing an intact skin and a wound environment although the AUC level may not reflect that (possibly due to high variance between samples).

Further analysis was performed to show the most predominant bacteria in intact skin and wound samples in Table 5. Several genera overlap between the distinguishing Table $\mathbf{4}$ and predominance Table 5, showing the bacterial genera composing majority of the samples. Chryseobacterium, Segetibacter and Methylophilus are seen in both Table $\mathbf{4}$ and Table 5, indicating some importance within intact skin samples.

Table 5 further shows that Escherichia spp. and Shigella spp. represented genera in wounds. E. coli are one of the most prevalent bacterial species in the surroundings. Although Escherichia were grouped with the least discriminating bacteria, possibly due to a high variance seen within wound samples as mentioned earlier, the wound samples within which the genus was found showed higher levels of Escherichia than intact skin samples. Furthermore, Escherichia and Shigella share many genes and have a similar genome [32], particularly in the amplification region being analyzed here. During this study, identification of tentative consensus (TC) sequences was performed using alignment principles. When the sequence in question was aligned to a sequence from our custom database, best alignments were considered. Table 6 shows all occurrences of Escherichia, Shigella, and Serratia, a genus also related to Escherichia and Shigella [33], and the counts found in both intact and wound samples. Bacteria belonging to the Escherichia, Shig- 
Table 5. 10 Most Prevalent Genera in Intact Skin and Wounds

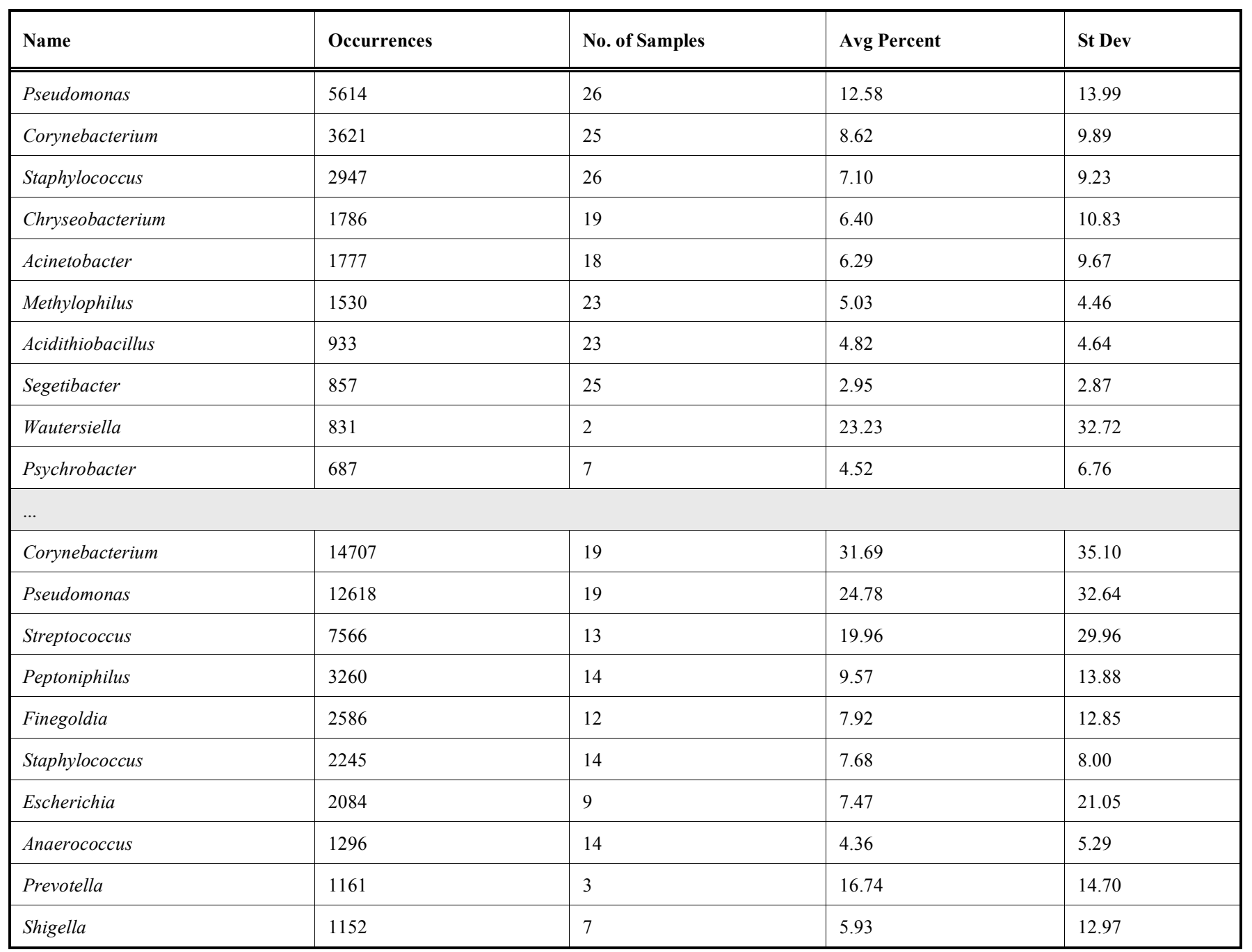

The first ten bacteria listed are the most prevalent genera found in intact skin and the last ten are the ten most prevalent genera in wound samples. The number of occurrences, the number of samples and the respective average percentages and standard deviation at which they were found are displayed.

Table 6. Escherichia, Shigella and Serratia Counts within Intact Skin and Wound Samples

\begin{tabular}{|l|c|c|c|c|c|c|}
\hline & \multicolumn{3}{|c|}{ Intact Skin } & & Wounds \\
\hline Bacterial Genus & Counts & No. of Samples & Avg Percent & Counts & No. of Samples & Avg Percent \\
\hline \hline Shigella & 14 & 7 & 0.84 & 1152 & 7 & 5.95 \\
\hline Serratia & 1 & 1 & 0.03 & 556 & 9 & 7.52 \\
\hline Escherichia & 31 & 11 & 0.66 & 2084 & 9.40 \\
\hline Sum & $\mathbf{4 6}$ & & & $\mathbf{3 7 9 2}$ & \\
\hline
\end{tabular}

Information for the three related genera with statistics at which they were encountered in intact skin and wounds are displayed.

ella and Serratia genera are often responsible for severe skin infections [34]. These values should all be considered due to the close relation among the genera and therefore possibly synonymous alignment and identification in the region analyzed. The frequency pattern for the three bacterial groups displays a strong favor to the wound samples, supporting the bacterial synonimity and importance.

\section{Comparison Analysis}

To ensure the existence of significant differences among skin and wound samples, Principal Component Analysis (PCA) was performed. Fig. (1) shows the resulting analysis for the principal components. The intact skin and wound groups clearly cluster, implying the bacterial diversity and 


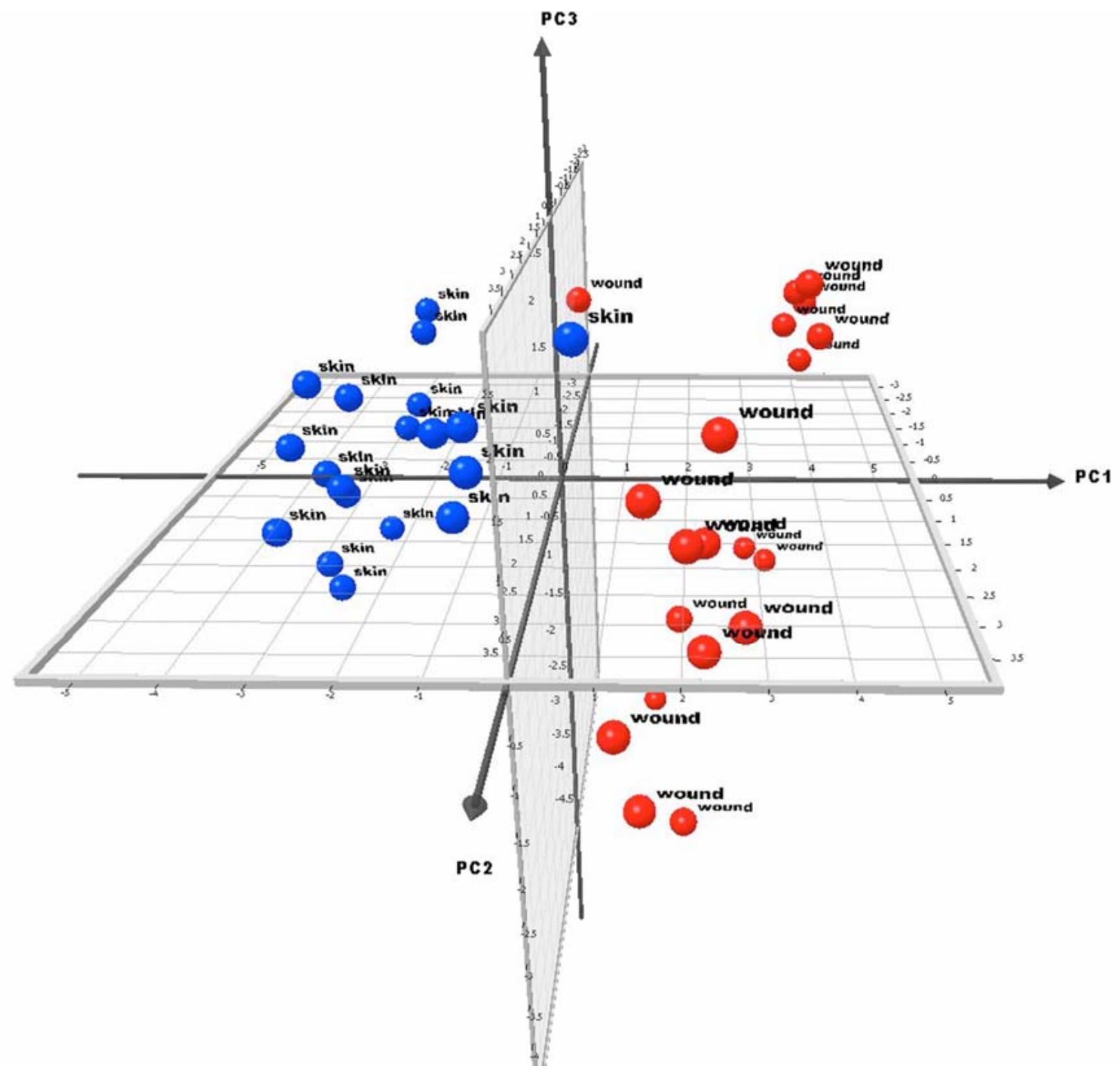

Fig. (1). PCA for intact skin and wound data. The figure display the three main principal components to which the data was reduced to. The axes represent the values for principal components 1, 2 and 3. Points lying in the negative portion of an axis indicate a negative correlation between the principal component and the sample. The two groups are denoted by different colors to demonstrate the separation between classes (intact skin vs. wounds). The ability to linearly separate the classes within the PCA figures indicates intact skin samples are different from wound samples.

microbial composition of the samples is different and unaffecting of each other. The PCA scores for the three principal components are displayed on the axes. Scores are results of a weight applied to the original data with the result indicating a negative or positive correlation with the component.

Hierarchical clustering was also performed on the data with the results shown in Fig. (2). The intact skin and wounds groups are separated at the top level, dividing the samples perfectly. The dendogram supports the previously discussed results indicating the ability to differentiate healthy skin and wounds based on bacterial composition.
Furthermore, Fig. (2) also demonstrates the lack of strong similarity between healthy skin and wounds for the same patient. The possibility of contamination is not statistically supported by the results demonstrated in this image.

To further investigate the similarity of healthy skin and wound samples from the same patient, samples with pairs for a corresponding part of the body for an individual were studied to evaluate the extent of similarity in the bacterial composition of the samples. A similarity measure matrix representing all possible pairs was created and can been seen in Fig. (3). The results of the analysis demonstrate no 


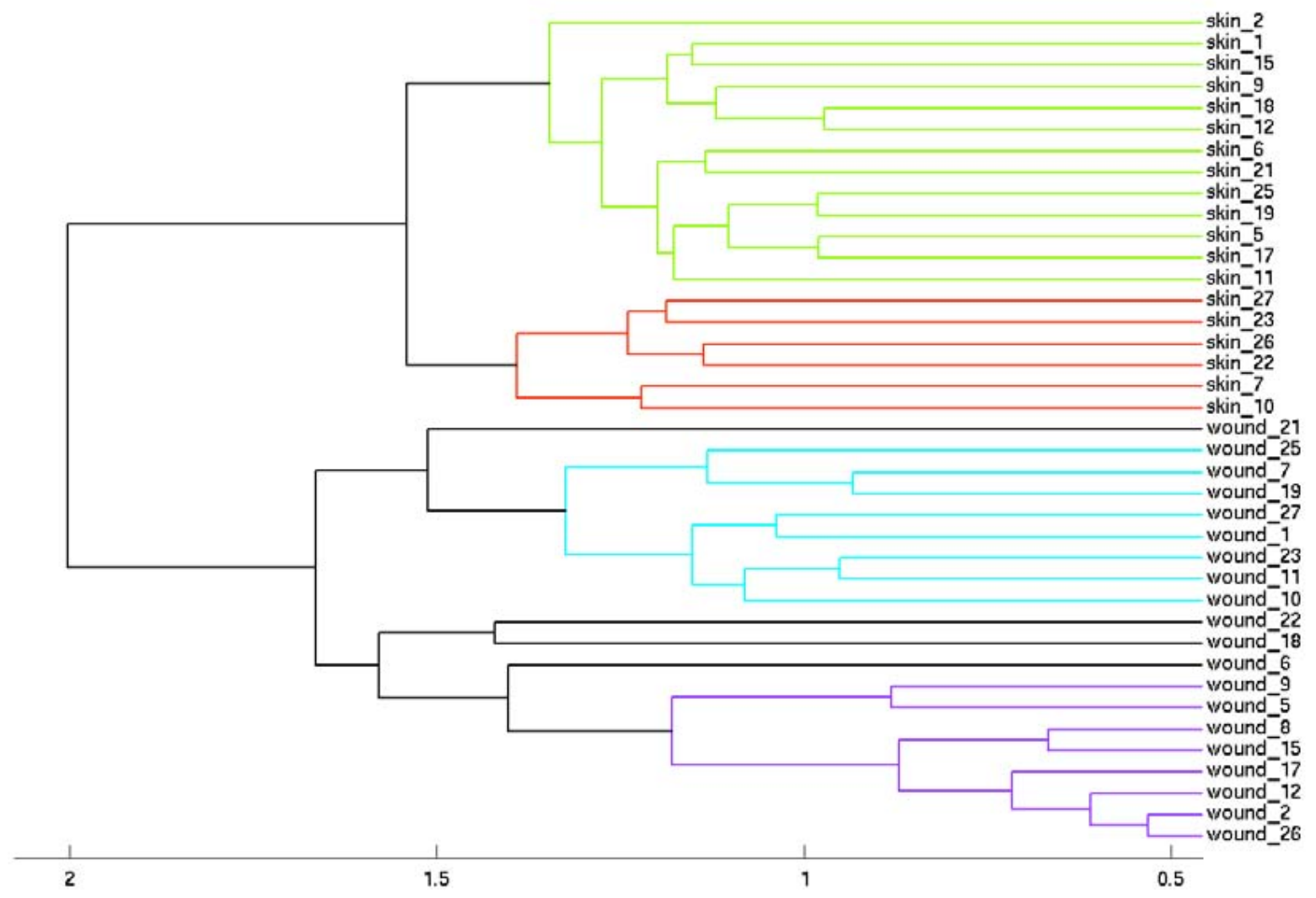

Fig. (2). Hierarchical clustering of healthy skin and wound data. This figure provides further support for the separability between the two classes of samples, healthy skin and wounds. The figure also indicates low correlation between healthy skin and wound samples for the same individual, indicating lack of contamination or other factors possibly affecting bacterial similarity.

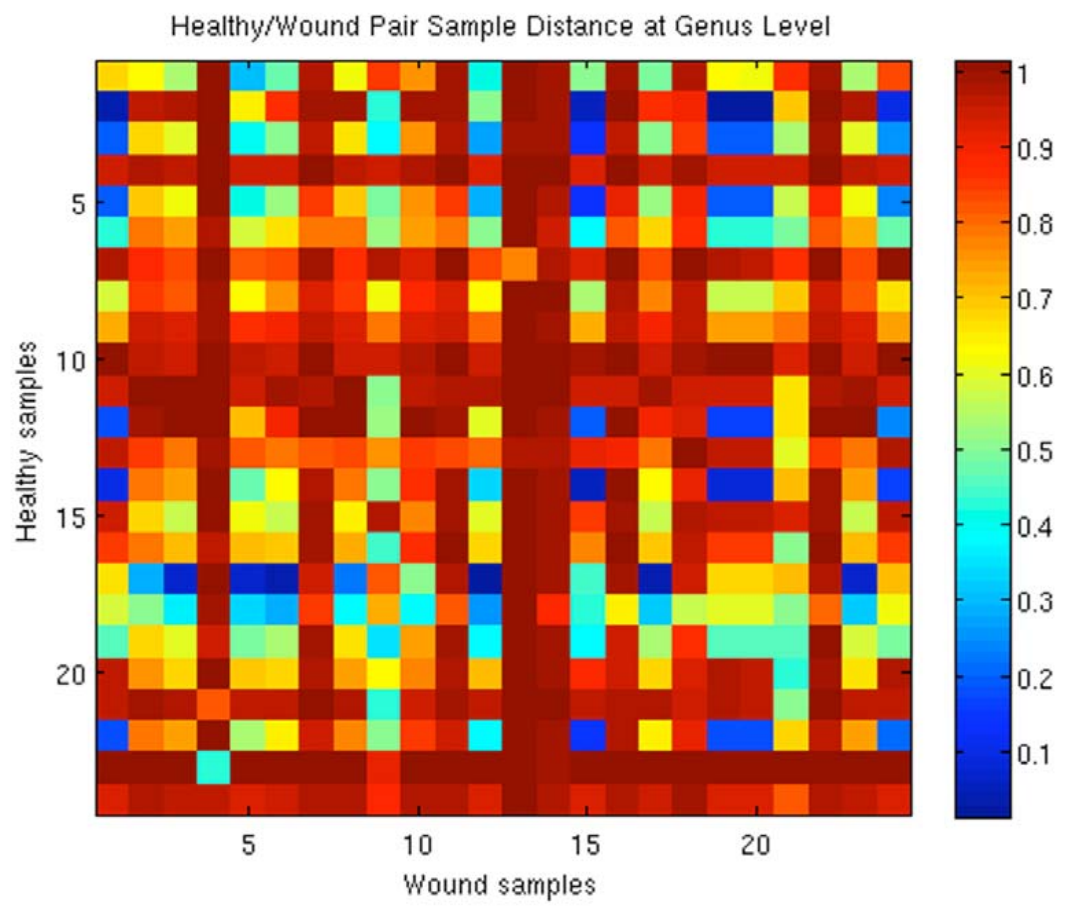

Fig. (3). Correlation Distances for paired intact skin against wound samples. Pearson Correlation distances, ranging from 0 to 2 were normalized to a scale of 0 to 1 where 1 represents the furthest distance, or the least similar samples. 
conclusive relationships between an individual's wound and intact skin samples. The image in Fig. (3) shows the relationship between each intact and wound sample based on the Pearson's correlation distance. A distance of zero represents the closest samples, while a distance of two (scaled to 1 for the image) is the furthest. A color spectrum is used to represent these distances, and the closest distances will be the coolest tones, closest to blue, while the furthest ones will be closer to the red colors. If the corresponding wound and intact skin samples were similar, indicating contamination or the flora of intact skin being strongly affected by the flora of the wounds (or vice versa), the blue color would be seen on the diagonal of the heat map matrix. Judgment should be made by complementary indices on the $\mathrm{x}$ and $\mathrm{y}$ axis, (i.e. sample 1 of the intact skin, corresponds to sample 1 of the wounds and the relationship between them can be seen in the top left corner of the matrix). Similarly, sample 2 would be in position $(2,2)$, and so on for the rest of the samples. The diagonal, however, is diverse in color, with the average Pearson's correlation distance of 0.78 , showing a relationship between intact skin and wounds in a corresponding location.

A distance analysis was also done on all against all samples, not limiting to corresponding pairs. Fig. (4) shows this data. Unlike Fig. (3), a blue diagonal line can be seen, it represent the distance between the same sample. This forms a logical deduction that sample 1 (whether wound or skin) is going to be identical to sample 1. Fig. (4) is useful for analysis of distances of skin vs. skin, skin vs. wound and wound vs. wound. Within the analysis, there were 28 intact samples and 24 wound samples. The $28 \times 28 \mathrm{NW}$ corner of the map is the distance within intact samples, $24 \times 24$ SE corner is within wound samples, and the rest is between intact and wound samples. The matrix is symmetric so essentially only the upper or lower triangle requires analysis. The sample comparison again does not display an obvious pattern, however, evaluating the NE corner of the figure, the intact/wound region, it is evident the area is primarily in the red tones further supporting the conclusions drawn and discussed in relation to Fig. (3). The correlation distance between all intact skin and wound samples is 0.79 . In the SE corner, however, the distances between the wound samples appear to be closer. There is much more blue seen in the figure, showing closer correlations between the wounds. The increased intensity of the red, however, offsets the closer relationships, resulting in an average correlation distance of 0.77 . The intact samples, in the NW corner, although do not have as much true blue tones as the wounds, have a greater amount of mid tones, also resulting in a closer correlation that the intact/wound comparisons. The average distance within intact skin samples is 0.70 , the closest of all three groups. Group analysis of intact against intact samples shows the lowest distance, suggesting the most similarity. The Pearson correlation distance is in the $[0,2]$ range, thus implying the distances of all three groups, being less than 1 , are closer than the average random correlation value.

\section{DISCUSSION}

\section{Diversity Analysis}

The bacterial microbiota analysis results indicate there is a significantly lower diversity of bacteria found in wounds than in intact skin. More sequences per sample were analyzed within wound data, intuitively indicating more diver-

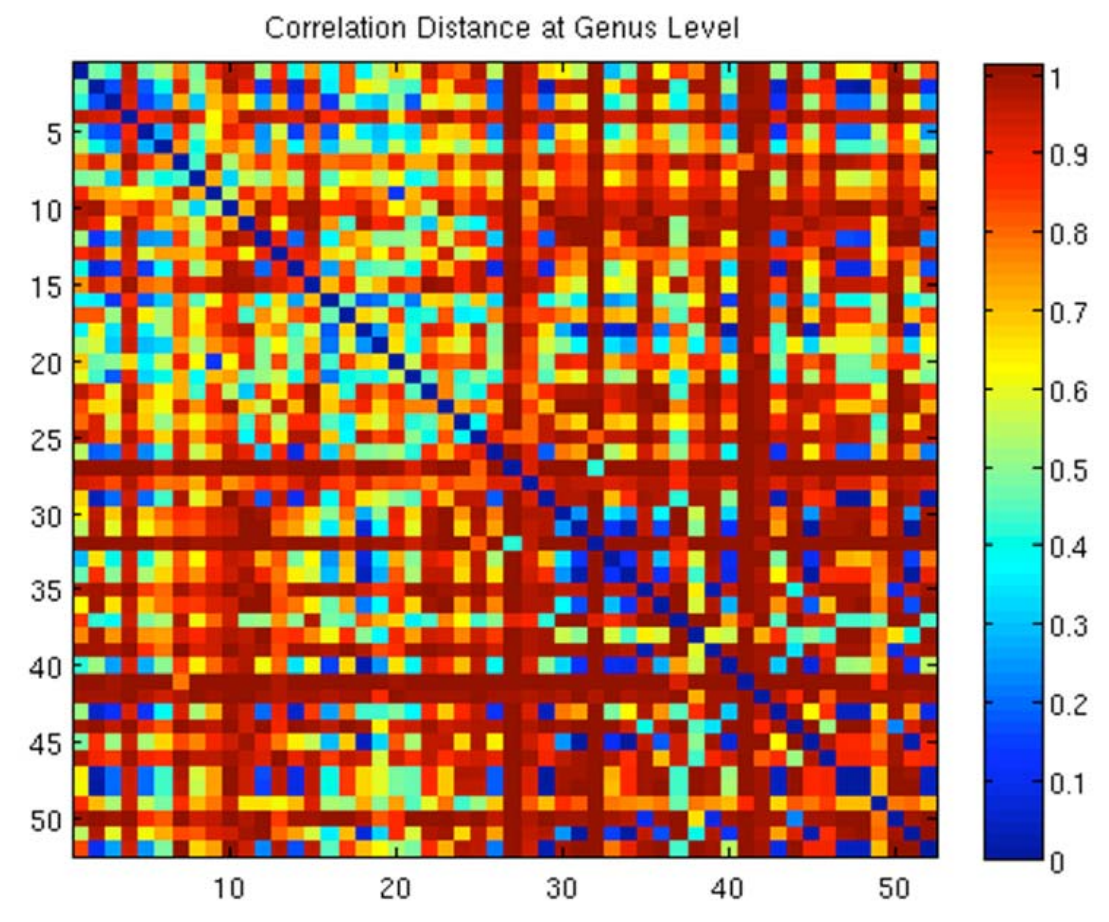

Fig. (4). Correlation Distances for all against all samples. Pearson Correlation distances, ranging from 0 to 2 were normalized to a scale of 0 to 1 where 1 represents the furthest distance, or the least similar samples. 
sity, however intact skin samples proved to be more diverse. This information, along with significance summaries can be seen in Table 3. There is not a multitude of information comparing healthy skin to wounds, however a number of studies have shown the overall diversity of healthy skin to be considerable [35-37]. The more abundant flora of intact skin may be contributing to its robust environment and protecting the ecosystem from the spread of infection or accumulation of opportunistic and pathogenic populations. Previous studies have indicated the properties of bacterial microflora of intact skin demonstrate advantages to the system [38, 39] and thus support the resistance in healthy skin and the vulnerability to complications witnessed in wounds. The type of diversity data presented within Tables 1-3 is not able to precisely indicate what diversifies the two sets of samples. However, this type of analysis is an established means of evaluating the overall species richness within groups [40].

\section{Composition Analysis}

Table 4 indicates bacteria falling into genera like Corynebacterium, Streptococcus and Anaerococcus have been shown to be associated with chronic wounds, and our results coincide with their findings. However, Table 4 also indicates intact skin to also contain Corynebacterium genera as part of the predominant composition. The percentages at which these bacteria are found, in wounded skin are higher indicating Corynebacterium as a significant opportunistic contributor to chronic wounds. Similarly, Streptococcus spp. are often associated with wounds and in this study the genus was only found in 17 of 29 intact skin samples, at an average of only $1.54 \%$ (data not shown), compared to $20 \%$ in wounds. This is evidence for elevated levels of certain bacteria normally indigenous potentially contributing to wound biofilm, bioburden and polymicrobial infection.

Returning to Corynebacterium, these bacteria are recognized as opportunistic human pathogens [41]. Corynebacterium genus harbors several species, including Corynebacterium jeikeium. This is a "lipophilic" and multidrug resistant bacterium of the human skin flora [42]. Corynebacterium jeikeium shares $69 \%$ of its genes with other species of the Corynebacterium genus [42]. This chromosomal backbone allows the bacteria to be toxic and prevalent and possibly drug resistant. This coincides with the fact and indicates the importance of the observation that such Corynebacterium were found at much higher levels in the wounds than intact skin samples.

Anaerobes have often been found in wound samples and are recognized as a major population in chronic wound biofilms [7, 43-47]. Genera like Finegoldia and Peptoniphilus are part of the anaerobic bacteria group and are some of the most common genera found in the wound samples of this study (Table 5). This data agrees with the previously discussed importance and prevalence of these and other anaerobes [43, 48-53]. Anaerobes have been shown to be a uniting force among various multi species groups found in chronic wound infections [43]. Although wounds are generally exposed to air [43], this group of bacteria may survive the harmful effects of oxygen by symbiotically existing with aerobic bacteria, a process known as co-aggregation [54, 55]. It has also been shown that oxygen does not reach the inter- nal regions of biofilms, leaving the harmful and prevalent anaerobes unaffected [56].

Several genera seen in Table 5, although are found to occur in the top ten bacterial genera, are found in few samples. Although Wautersiella spp. and Prevotella spp. are prevalent in intact skin and wound samples, respectively, they are not predominant within those samples. Because so few samples contain the genera, little can be said about their importance.

Table 6 addresses the E. coli composition within wounds. Although humans generally remain unaffected by $E$. coli presence, opportunistically $E$. coli can cause serious infections. Intact skin can act as a barrier from $E$. coli infection. Due to a binding protein present at the surface of the skin, $E$. coli is unable to survive and colonize [57]. It is evident the wounds have significantly greater bacterial numbers and work is well underway to elucidate these anecdotal observations. Contributing to this is the absence of functional skin barriers, exposed tissue, and therefore in the absence of psoriasin, the binding protein protecting skin, E. coli is able to colonize and grow on the wounds.

\section{Comparison Analysis}

The similarity analysis performed on the data using Pearson's methodologies resulted in supportive results for the intact skin and wound samples being somewhat similar. When the analysis was performed on paired samples, specimens extracted from the same individual in corresponding locations (for example a wound on the right ankle and intact skin on the left ankle), the correlation distance was 0.78 , while the comparison on unpaired intact/wound samples results in 0.79 . Because the paired and unpaired statistics are so close in value, it cannot be concluded that the intact or wounded skin affects the other. Although this number indicates a similarity on a scale of 0 to 2 the average, random distance between any two samples statistically should be 1 , it cannot be concluded that an individual wound should be more similar to the corresponding intact location than it should be to any other person's intact skin. Because the Pearson's correlation distance we are dealing with is less than 1 , this could imply that the flora of the wound may affect that of the intact skin, or it may be just an artifact of diabetic human flora.

The Pearson's correlation values from the unpaired analysis indicate that intact skin shares the most similarity with other intact skin. A similar statement can be made for wounds. The statistics indicate that although the differentiation can be made by a small margin, intact skin and wound samples are less similar to each other than to other samples within their group. Ultimately we find that the microbiome of chronic wounds is very distinct in composition when comparing intact skin and chronic wounds. It is obvious that the chronic wound environment promotes propagation and accumulations of key opportunistic pathogenic populations supporting the concepts of functionally equivalent pathogroups. Work will continue to more fully understand the microbiome of skin and wounds including efforts to elucidate alternative strategies and hypotheses such as the potentials of probiotic microbiota that may promote wound healing. 


\section{ACKNOWLEDGEMENT}

Funding: The study was funded by internal research monies of Medical Biofilm Research Institute. Mention of trade names does not represent endorsement by the authors of this work.

\section{REFERENCES}

[1] Bjarnsholt T, Kirketerp-Moller K, Jensen PO, et al. Why chronic wounds will not heal: a novel hypothesis. Wound Repair Regen 2008; 16: 2-10.

[2] Costerton JW, Lewandowski Z, Caldwell DE, Korber DR, LappinScott HM. Microbial biofilms. Annu Rev Microbiol 1995; 49: 71145.

[3] Wolcott RD, Rhoads DD. A study of biofilm-based wound management in subjects with critical limb ischaemia. J Wound Care 2008; 17: 145-2, 154.

[4] James GA, Swogger E, Wolcott R, et al. Biofilms in chronic wounds. Wound Repair Regen 2008; 16: 37-44.

[5] Percival SL, Bowler P, Woods EJ. Assessing the effect of an antimicrobial wound dressing on biofilms. Wound Repair Regen 2008; 16: 52-7.

[6] Wolcott RD, Ehrlich GD. Biofilms and chronic infections. JAMA 2008; 299: 2682-4.

[7] Dowd SE, Sun Y, Secor PR, et al. Survey of bacterial diversity in chronic wounds using pyrosequencing, DGGE, and full ribosome shotgun sequencing. BMC Microbiol 2008; 8: 43.

[8] Wolcott RD, Kennedy JP, Dowd SE. Regular debridement is the main tool for maintaining a healthy wound bed in most chronic wounds. J Wound Care 2009; 18: 54-6.

[9] CDCDFWC: Consensus Development Conference on Diabetic Foot Wound Care. Boston, Massachusetts: American Diabetes Association 1999; pp. 1354-1360.

[10] National Diabetes Information Clearinghouse. National Diabetes Statistics 2007. Ref Type: Online Source

[11] Ismail K, Winkley K, Stahl D, Chalder T, Edmonds M. A cohort study of people with diabetes and their first foot ulcer: the role of depression on mortality. Diabetes Care 2007; 30: 1473-9.

[12] MMWR. History of foot ulcer among persons with diabetes-United States, 2000-2002. MMWR Morb Mortal Wkly Rep 2003; 52: 1098-102.

[13] Wolcott RD, Gontcharova V, Sun Y, Zischkau AM, Dowd SE. Bacterial diversity in surgical site infections: not just aerobic cocci any more. J Wound Care 2009; 18: 317-23.

[14] Dowd SE, Wolcott RD, Sun Y, McKeehan T, Smith E, Rhoads D. Polymicrobial nature of chronic diabetic foot ulcer biofilm infections determined using bacterial tag encoded FLX amplicon pyrosequencing (bTEFAP). PLoS ONE 2008; 3: e3326.

[15] Rowan NJ. Viable but non-culturable forms of food and waterborne bacteria: Quo Vadis? Trends Food Sci Technol 2004; 15: 462.

[16] Wolcott RD, Dowd SE. A rapid molecular method for characterising bacterial bioburden in chronic wounds. J Wound Care 2008; 17: 513-6.

[17] Altschul SF, Gish W, Miller W, Myers EW, Lipman DJ. Basic local alignment search tool. J Mol Biol 1990; 215: 403-10.

[18] Hand DJ, Till RJ. A Simple Generalisation of the Area Under the ROC Curve for Multiple Class Classification Problems. Mach Learn 2001; 45: 171-86.

[19] Fawcett T. An introduction to ROC analysis. Pattern Recogn Lett 2006; 27: 861-74.

[20] Theodoridis S, Koutroumbas K. Pattern Recognition, $2^{\text {nd }}$ Ed. San Diego, Ca: Academic Press 2008.

[21] Stigler SM. Francis Galton's Account of the Invention of Correlation. Stat Sci 1989; 4: 73-86.

[22] Rodgers JL, Nicewander WA. Thirteen ways to look at the correlation coefficient. Am Stat 1988; 42: 59-66.

[23] Lozupone C, Hamady M, Knight R. UniFrac--an online tool for comparing microbial community diversity in a phylogenetic context. BMC Bioinformatics 2006; 7: 371.

[24] Wolcott RD, Rhoads DD, Dowd SE. Biofilms and chronic wound inflammation. J Wound Care 2008; 17: 333-41.
[25] Finkel SE, Kolter R. Evolution of microbial diversity during prolonged starvation. Proc Natl Acad Sci USA 1999; 96: 4023-7.

[26] Hong SH, Bunge J, Jeon SO, Epstein SS. Predicting microbial species richness. Proc Natl Acad Sci USA 2006; 103: 117-22.

[27] Ptacnik R, Solimini AG, Andersen T, et al. Diversity predicts stability and resource use efficiency in natural phytoplankton communities. Proc Natl Acad Sci USA 2008; 105: 5134-8.

[28] Sogin ML, Morrison HG, Huber JA, et al. Microbial diversity in the deep sea and the underexplored "rare biosphere". Proc Natl Acad Sci USA 2006; 103: 12115-20.

[29] Schloss PD, Handelsman J. Introducing DOTUR, a computer program for defining operational taxonomic units and estimating species richness. Appl Environ Microbiol 2005; 71: 1501-6.

[30] Dowd SE, Callaway TR, Wolcott RD, et al. Evaluation of the bacterial diversity in the feces of cattle using 16S rDNA bacterial tag-encoded FLX amplicon pyrosequencing (bTEFAP). BMC Microbiol 2008; 8: 125.

[31] Chao A, Bunge J. Estimating the number of species in a stochastic abundance model. Biometrics 2002; 58: 531-9.

[32] Brenner DJ, Fanning GR, Skerman FJ, Falkow S. Polynucleotide sequence divergence among strains of Escherichia coli and closely related organisms. J Bacteriol 1972; 109: 953-65.

[33] Ohnishi Y, Beppu T, Horinouchi S. Two genes encoding serine protease homologues in Serratia marcescens and characterization of their products in Escherichia coli. J Biochem 1997; 121: 902-13.

[34] Langrock ML, Linde HJ, Landthaler M, Karrer S. Leg ulcers and abscesses caused by Serratia marcescens. Eur J Dermatol 2008; 18: 705-7.

[35] Dekio I, Hayashi H, Sakamoto M, et al. Detection of potentially novel bacterial components of the human skin microbiota using culture-independent molecular profiling. J Med Microbiol 2005; 54: 1231-8.

[36] Bek-Thomsen M, Lomholt HB, Kilian M. Acne is not associated with yet-uncultured bacteria. J Clin Microbiol 2008; 46: 3355-60

[37] Gao Z, Tseng CH, Pei Z, Blaser MJ. Molecular analysis of human forearm superficial skin bacterial biota. Proc Natl Acad Sci USA 2007; 104: 2927-32.

[38] Roth RR, James WD. Microbial ecology of the skin. Annu Rev Microbiol 1988; 42: 441-64.

[39] Krutmann J. Pre- and probiotics for human skin. J Dermatol Sci 2009; 54: 1-5.

[40] Raup DM. Taxonomic diversity estimation using rarefaction. Paleobiology 1975; 1: 333-42.

[41] Funke G, Von GA, Clarridge JE, III, Bernard KA. Clinical microbiology of coryneform bacteria. Clin Microbiol Rev 1997; 10: $125-59$

[42] Tauch A, Kaiser O, Hain $\mathrm{T}$, et al. Complete genome sequence and analysis of the multiresistant nosocomial pathogen Corynebacterium jeikeium K411, a lipid-requiring bacterium of the human skin flora. J Bacteriol 2005; 187: 4671-82.

[43] Bowler PG, Davies BJ. The microbiology of infected and noninfected leg ulcers. Int J Dermatol 1999; 38: 573-8.

[44] Brook I, Frazier EH. Aerobic and anaerobic microbiology of chronic venous ulcers. Int J Dermatol 1998; 37(6): 426-8.

[45] Brook I. Role of encapsulated anaerobic bacteria in synergistic infections. Crit Rev Microbiol 1987; 14: 171-93.

[46] Mayrand D, McBride BC. Exological relationships of bacteria involved in a simple, mixed anaerobic infection. Infect Immun 1980; 27: 44-50.

[47] Urbancic-Rovan V, Gubina M. Bacteria in superficial diabetic foot ulcers. Diabet Med 2000; 17: 814-5.

[48] Trengove NJ, Stacey MC, McGechie DF, Mata S. Qualitative bacteriology and leg ulcer healing. J Wound Care 1996; 5: $277-$ 80 .

[49] Bowler PG, Davies BJ, Jones SA. Microbial involvement in chronic wound malodour. J Wound Care 1999; 8: 216-8.

[50] Hansson C, Hoborn J, Moller A, Swanbeck G. The microbial flora in venous leg ulcers without clinical signs of infection. Repeated culture using a validated standardised microbiological technique. Acta Derm Venereol 1995; 75: 24-30.

[51] Kontiainen S, Rinne E. Bacteria in decubitus ulcers. Infection 1988; 16: 305 . 
[52] Kontiainen S, Rinne E. Bacteria in ulcera crurum. Acta Derm Venereol 1988; 68: 240-4.

[53] Howell-Jones RS, Wilson MJ, Hill KE, et al. A review of the microbiology, antibiotic usage and resistance in chronic skin wounds. J Antimicrob Chemother 2005; 55: 143-9.

[54] Bradshaw DJ, Marsh PD, Watson GK, Allison C. Role of Fusobacterium nucleatum and coaggregation in anaerobe survival in planktonic and biofilm oral microbial communities during aeration. Infect Immun 1998; 66: 4729-32.
[55] Bradshaw DJ, Marsh PD, Allison C, Schilling KM. Effect of oxygen, inoculum composition and flow rate on development of mixed-culture oral biofilms. Microbiology 1996; 142: 623-9.

[56] Rasmussen K, Lewandowski Z. Microelectrode measurements of local mass transport rates in heterogeneous biofilms. Biotechnol Bioeng 1998; 59: 302-9.

[57] Bevins CL. An important clue: fingerprints point to psoriasin in defense against E. coli. Nat Immunol 2005; 6: 12-3.

Received: February 15, 2010

Revised: February 22, 2010

Accepted: February 26, 2010

(c) Gontcharova et al.; Licensee Bentham Open.

This is an open access article licensed under the terms of the Creative Commons Attribution Non-Commercial License (http://creativecommons.org/licenses/ by-nc/3.0/) which permits unrestricted, non-commercial use, distribution and reproduction in any medium, provided the work is properly cited. 